\title{
El uso del sildenafil en pacientes con enfermedad coronaria no aumentó el riesgo de isquemia del miocardio
}

Cardiovascular Effects of Sildenafil During Exercise in Men With Known or Probable Coronary Artery Disease. Arruda - Olson A, Mahoney D, Nehra A y col. JAMA. 2002 Feb 13; 287 (6): 719-725.

\section{Objetivo}

Determinar los efectos cardiovasculares del sildenafil durante el ejercicio, en hombres con enfermedad coronaria o alta probabilidad de enfermedad coronaria.

Diseño

Ensayo clínico aleatorizado, doble ciego, controlado con placebo, cruzado*

Lugar

Centro de cuidados ambulatorios en Rochester, Minnesota, EE.UU.

\section{Pacientes}

Hombres mayores de 40 años con Disfunción Sexual Eréctil (DSE) y cualquiera de los siguientes: 1) Enfermedad coronaria conocida: $>50 \%$ de estenosis de un vaso mayor o una de sus ramas mayores; historia de IAM; resultado positivo de un test por imagen; bypass 0 angioplastia.2) Probabilidad pretest de enfermedad coronaria $>70 \%$ en pacientes con angina de pecho. Los criterios de exclusión fueron: asma, enfermedad cardíaca inestable, insuficiencia hepática, renal y tensión arterial (TA) sistólica $<90$ $\mathrm{MmHg}$. Se incluyeron 110 pacientes, ninguno de ellos había recibido tratamiento con dipiridamol, teofilina, eritromicina, cimetidina o sildenafil en las últimas 24 hs.

\section{Intervención}

Se aleatorizaron 110 pacientes. La mitad recibió primero sildenafil (50 o 100mg) y luego placebo y la otra mitad recibió el tratamiento en orden inverso.Todos realizaban dos eco-estréss en iguales condiciones con un intervalo de uno a tres días. Una hora antes del test tomaban placebo o droga según la aleatorización. Si el paciente tomaba nitritos se le discontinuaban 72 hs antes. Los criterios para concluir el eco- estrés eran: desarrollo de síntomas (incluido fatiga), disminución de la TA sistólica $>10 \mathrm{MmHg}$, dilatación ventricular, reducción global de la función sistólica o arritmia significativa. El test era interpretado por un revisor ciego a otra información clínica.

\section{Medición de Resultados Principales}

Efectos hemodinámicos y cardiovasculares del sildenafil durante el ejercicio, determinados durante la realización del Eco-estrés.

\section{Resultados Principales}

No hubo diferencias significativas entre ningún grupo en la frecuencia cardíaca, la TA diastólica y el "índice de movimiento de pared" (IMP, una medida de extensión y severidad de anormalidades de movimiento de la pared cardiaca). Después de administrar el sildenafil la TA sistólica se redujo en promedio 7 $\mathrm{mmHg}$ (IC 95\%, -9 a -4 mmHg; $\mathrm{P}<0,001)$; después del placebo la reducción fue de $2 \mathrm{mmHg}$ (IC95\% -6 a $-0,3 \mathrm{mmHg} ; \mathrm{P}=0,08)$. Disnea o angina fueron reportadas por 69 pacientes en el grupo sildenafil y en 70 del grupo placebo $(P=N S)$. El Eco-estrés fue anormal en 84 pacientes luego de dar sildenafil y en 86 luego de dar placebo. $(\mathrm{P}=\mathrm{NS})$

\section{Conclusiones}

En pacientes con enfermedad coronaria sin uso de nitritos, el sildenafil no potencia la isquemia del miocardio.

\section{Comentario}

Se calcula una prevalencia de la DSE en EE.UU.del $35 \%$ a los 40 años y del $67 \%$ a los $70 .{ }^{1}$ Debido a la alta prevalencia de enfermedad coronaria al avanzar la edad, no es infrecuente que ambas coexistan. Trabajos previos establecen que el sildenafil tiene una efectividad cercana al $70 \%{ }^{2}$ y es una droga de manejo del médico de atención primaria.

Los efectos adversos cardiovasculares reportados incluyen IAM, taquicardia ventricular, hipotensión y muerte.Es probable que estos eventos se produzcan al interactuar con nitritos, incluso $24 \mathrm{hs}$ después de la toma del sildenafil, por lo cual su combinación está contraindicada. El presente trabajo evalúa la función cardíaca durante el ejercicio con una única toma del sildenafil a corto plazo;todavía faltarían estudios que evaluen los efectos del sildenafil a más largo plazo.

Existe una gran preocupación de prescribir sildenafil a pacientes con enfermedad coronaria. Este trabajo reasegura la indicación de la droga, sugiriendo que la isquemia y los eventos cardíacos reportados previamente están mayormente relacionados al uso de nitritos o al desgaste físico durante la actividad sexual más que al uso propio de la droga.

La única diferencia estadisticamente significativa encontrada en este estudio entre el grupo placebo y el sildenafil se refiere a la disminución de $7 \mathrm{mmHg}$ de la TA sistólica al tomar la droga, cuya significancia clínica es dudosa.

En pacientes coronarios, también es posible utilizar otros recursos farmacológicos como la apomorfina y la fentolamina (incluso en forma conjugada), que no reportan efectos cardiovasculares ni interacción con nitritos. ${ }^{4,5}$

Conclusiones del comentador: en pacientes con DSE y enfermedad coronaria, que no estén recibiendo nitritos, el sildenafil parece ser seguro y efectivo. En pacientes bajo tratamiento con nitritos, habría algunas opciones alternativas de tratamiento.

\section{Dr. Esteban Kuten [ Unidad de Medicina Familiar y Preventiva. Hospital Italiano de Buenos Aires ]}

\footnotetext{
Referencias

1. Rubinstein E.Disfunción Sexual eréctil.Medicina Familiar y Práctica Ambulatoria.2001, 40;298 -304. Editorial Panamericana.

2. Rubinstein E.Disfunción Sexual eréctil.Medicina Familiar y Práctica Ambulatoria.2001, 40;298 -304. Editorial Panamericana. 202 Jun 24:162 (12):1349-60

3.Marwick, T. Safe Sex for Men With Coronary Artery Disease: Exercise, Sildenafil, and Risk of Cardiac Events.J AMA,2002. Feb 13, 287 (6): 766-767.

4. Lammers P., Rubio-Aurioles E, Castell R.Combination Therapy for erectile dysfunction:a randomized, double blind, unblinded active-controlled, cross-over study of the pharmacodynamics and safety of combined oral formulations of apomorphine hydrochloride, phentolamine mesylate and papaverine hydrochloride in men with moderate to severe erectile dysfunction. Int $\mathrm{J}$ Impot Res 2002 Feb; 14 (1):54-9.

5.Von Keitz A, Stroberg P, Bukofzer S.A European multicentre study to evaluate tolerability of apomorphine subligual administered in a forced dose escalation regimen in patients with erectile dysfunction. BJU Int 2002 Mar;89 (4): 409-415
} 